Article

\title{
Optical Fiber for High-Power Optical Communication
}

\author{
Kenji Kurokawa
}

NTT Access Network Service Systems Laboratories, 1-7-1 Hanabatake, Tsukuba 305-0805, Japan; E-Mail: kurokawa.kenji@lab.ntt.co.jp; Tel.: +81-29-868-6435; Fax: +81-29-868-6440

Received: 27 July 2012; in revised form: 6 September 2012 / Accepted: 10 September 2012 /

Published: 28 September 2012

\begin{abstract}
We examined optical fibers suitable for avoiding such problems as the fiber fuse phenomenon and failures at bends with a high power input. We found that the threshold power for fiber fuse propagation in photonic crystal fiber (PCF) and hole-assisted fiber (HAF) can exceed $18 \mathrm{~W}$, which is more than 10 times that in conventional single-mode fiber (SMF). We considered this high threshold power in PCF and HAF to be caused by a jet of high temperature fluid penetrating the air holes. We showed examples of two kinds of failures at bends in conventional SMF when the input power was $9 \mathrm{~W}$. We also observed the generation of a fiber fuse under a condition that caused a bend-loss induced failure. We showed that one solution for the failures at bends is to use optical fibers with a low bending loss such as PCF and HAF. Therefore, we consider PCF and HAF to be attractive solutions to the problems of the fiber fuse phenomenon and failures at bends with a high power input.
\end{abstract}

Keywords: photonic crystal fiber; hole-assisted fiber; fiber fuse; high-power

\section{Introduction}

The optical power levels used in optical communication networks have been increasing with the development of long unrepeatered submarine systems, dense wavelength-division-multiplexing (WDM) systems, and distributed Raman amplification systems. Furthermore, the rapid growth in Internet traffic has led to a huge demand for transmission capacity. If we are to meet this exponential demand for capacity, we must greatly increase the optical transmission power. Thus there is a growing concern about the impact of high optical power on fiber reliability. It has been reported that high optical power can lead to catastrophic damage as a result of the optical fiber fuse phenomenon [1,2] or in tightly bent fibers [3]. 
A fiber fuse is an optical discharge propagating toward a light source that results in the catastrophic destruction of an optical fiber. Once initiated, the fiber fuse continues to propagate until the light source is shut down or the input power is reduced below the fuse-propagation threshold power. The propagation threshold of a fiber fuse in conventional fiber is as low as 1.2-1.4 W [4]. Therefore, in the near future the fiber fuse phenomenon will pose a real danger to optical communication systems constructed with conventional single-mode fibers [5]. There have already been many experimental and theoretical reports on the fiber fuse phenomenon [6-21]. In addition, several devices have been proposed with a view to avoiding the catastrophic damage caused by a fiber fuse, for example, a fiber fuse terminator using a tapered fiber [22] and a thermally-diffused expanded core (TEC) fiber [23]. A device has been reported that can rapidly detect a fiber fuse and terminate it by monitoring the light backreflected from it [24].

Recently, it was reported that the fuse propagation threshold in photonic crystal fiber (PCF) [10] and hole-assisted fiber (HAF) [18,20] can be much higher than that in conventional single-mode fiber (SMF) in the optical communication band. PCFs are attractive as transmission media with a broad bandwidth since they have unique features unavailable with conventional single-mode fiber (SMF) such as endlessly single-mode operation [25]. We have reported many applications of low loss PCF as a transmission medium [26]. HAFs are attractive as a bend-insensitive fiber [27] and are used in indoor optical cable.

Failures at bends are caused by the light that leaks from the core when the fiber is accidentally tightly bent under high power [3,28-37]. The fiber coating absorbs the leaked light and its temperature increases, and a failure occurs at the bend. Two kinds of serious failures at bends have been reported by Sikora et al., which they named regime 1 (R1) and regime 2 (R2) failures [29]. They are described as follows.

R1 failure: Catastrophic fiber failure within a few hours. The softening temperature of silica is reached $\left(~ \approx \approx 1100{ }^{\circ} \mathrm{C}\right.$ ) causing it to deform under stress. The pump power is no longer transmitted through the fiber.

R2 failure: Substantial oxidation of the fiber coating within a few days. The coating is burned off or vaporized but the silica remains largely intact. Subsequent handling or local disturbances will cause the fiber to fracture.

Since failures at bends will lead to the catastrophic failure of the fiber and burning of the coating, we must find a solution to the problem.

In this paper, we examined optical fibers that allow us to avoid such problems as the fiber fuse phenomenon and failures at bends when the input power is high. First, we found that the threshold power for fiber fuse propagation in PCF and HAF can exceed $18 \mathrm{~W}$, which is more than 10 times that in conventional SMF. We observed the termination dynamics of the fiber fuse near a splice point between a test fiber (PCF or HAF) and a conventional single-mode fiber. We considered that a fiber fuse in PCF and HAF was terminated by a jet of high temperature fluid penetrating the air holes. Second, we showed examples of two kinds of failures at bends (R1 and R2 failures) in conventional SMF when the input power was $9 \mathrm{~W}$ at a wavelength of $1480 \mathrm{~nm}$. We also observed the generation of a fiber fuse when the bend diameter was $13 \mathrm{~mm}$, which corresponded to an R1 failure. We describe reported solutions for the problems of the failures at bends. We show that one of the solutions involves using optical fibers with a low bending loss such as PCF and HAF. In conclusion, we consider that 
PCF and HAF are attractive optical fibers that can solve the problems presented by the fiber fuse phenomenon and failures at bends when the input power is high.

\section{Termination of Fiber Fuse Propagation in Photonic Crystal Fiber (PCF) and Hole-Assisted Fiber (HAF)}

\subsection{Fiber Parameters and Experimental Setup}

Figure 1 shows cross-sections of (a) PCF, (b) HAF, and (c) conventional SMF for comparison. We used a PCF with 60 air holes. Its structural parameter $d / \Lambda$ was 0.54 . Here, $d$ and $\Lambda$ are the hole diameter and hole pitch, respectively. $\Lambda$ was $5.7 \mu \mathrm{m}$. We used a HAF with six air holes. The core diameter was $9 \mu \mathrm{m}$. The diameter of an inscribed circle linking the air holes $c$ and the hole diameter $d$ were 17.1 and $4.6 \mu \mathrm{m}$, respectively.

Figure 1. Cross-Sections of (a) Photonic Crystal Fiber (PCF); (b) Hole-Assisted Fiber (HAF); and (c) conventional Single-Mode Fiber (SMF).
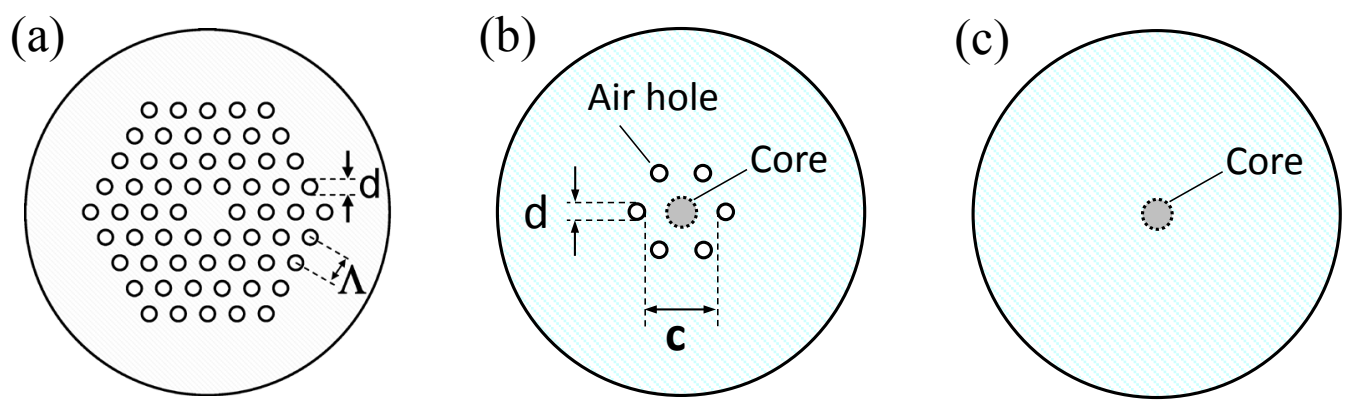

Figure 2 shows our experimental setup. The light sources used for the initiation and propagation of the fiber fuse were a Raman fiber laser and an amplified ASE (amplified spontaneous emission) operating at wavelengths of 1480 and $1550 \mathrm{~nm}$, respectively. The amplified ASE was obtained using an ASE light source, band-pass filters (BPFs), and erbium-doped optical fiber amplifiers (EDFAs). Two cw lights were simultaneously multiplexed with a WDM coupler to obtain a high input power of more than $10 \mathrm{~W}$, and then guided into a test fiber after passing through fiber 1 (dispersion-shifted fiber (DSF) or SMF). We initiated a fiber fuse by heating fiber 2 (DSF or SMF) with an arc discharge, and we examined its propagation in the test fiber. We observed the dynamics of fiber fuse termination at a splice point between fiber 2 and a test fiber using a high-speed camera. 
Figure 2. Experimental setup.

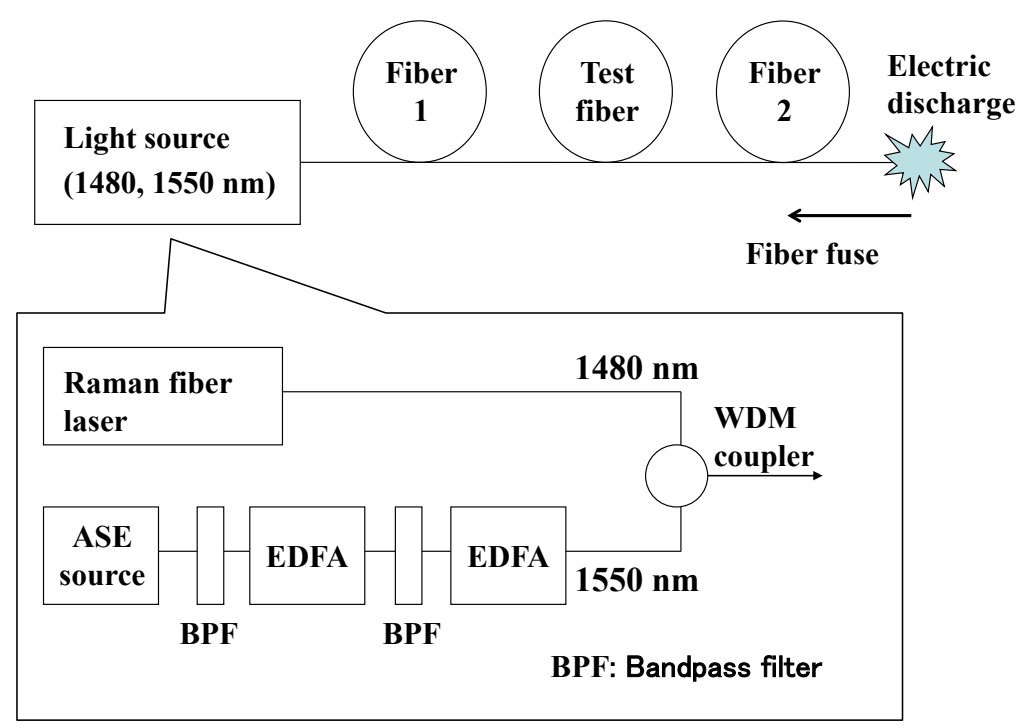

\subsection{Results and Discussion}

When the test fiber was a PCF, the fiber fuse propagation terminated at the splice point between fiber 2 (DSF) and the PCF even when the coupled input power into the PCF was at its maximum value of $18.1 \mathrm{~W}(6.6 \mathrm{~W}$ at $1480 \mathrm{~nm}$ and $11.5 \mathrm{~W}$ at $1550 \mathrm{~nm})$. Moreover, the fiber fuse did not propagate in the PCF when we heated it directly with an arc discharge but did not use fiber 2 . Since the propagation threshold of a fiber fuse is proportional to a mode field diameter [4], the threshold power in a PCF at $1550 \mathrm{~nm}$ is higher than that at $1480 \mathrm{~nm}$. Therefore, we can consider that the propagation threshold of a fiber fuse in this PCF is above $18.1 \mathrm{~W}$ at around $1550 \mathrm{~nm}$. This threshold power is more than 10 times higher than that in conventional SMF.

Figure 3 consists of four successive pictures taken at intervals of $0.1 \mathrm{~ms}$ that show the dynamics of fiber fuse termination at a splice point between a DSF and a PCF. The input power was $18.1 \mathrm{~W}$. The exposure time of the high-speed camera was $2.3 \mu \mathrm{s}$. As shown in Figure 3a, the optical discharge propagated in the DSF leaving periodic voids. When the front of the optical discharge reached the splice point, the front shape of the optical discharge changed as shown in Figure 3b. Since the optical discharge is a high temperature fluid [2,16], we consider that its front shape shown in Figure $3 b$ indicates a jet of high temperature fluid that penetrates the air holes of the PCF [38]. After $0.1 \mathrm{~ms}$ the optical discharge became small as shown in Figure 3c, and then terminated as shown in Figure 3d. In Figure 3c, we can also see the distinguishing front part of the jet in the PCF. We believe that we can explain the mechanism of the fiber fuse termination in PCF as follows. The size of the optical discharge is assumed to be almost the same as the diameter of the melted area $\left(D_{\text {melted }}\right)[18,20]$. The melted area is caused by a fiber fuse. The diameter of an inscribed circle in the air holes in the first layer of the PCF, which corresponds to parameter $c$ in HAF, was $8.3 \mu \mathrm{m}$, and it was much smaller than the measured $D_{\text {melted }}$ value of $18.3 \mu \mathrm{m}$ in the DSF. Therefore, the high temperature fluid reached the air holes, when the optical discharge penetrated the PCF. At the same time, the jet of fluid penetrated the air holes. That is, the high temperature fluid expanded. This resulted in a reduction in the fluid pressure and its temperature. Then, the fiber fuse terminated [38]. 
Figure 3. Dynamics of fiber fuse termination near the splice point between PCF and Dispersion-Shifted Fiber (DSF). (a)-(d) were obtained at intervals of $0.1 \mathrm{~ms}$. Exposure time was $2.3 \mu \mathrm{s}$.

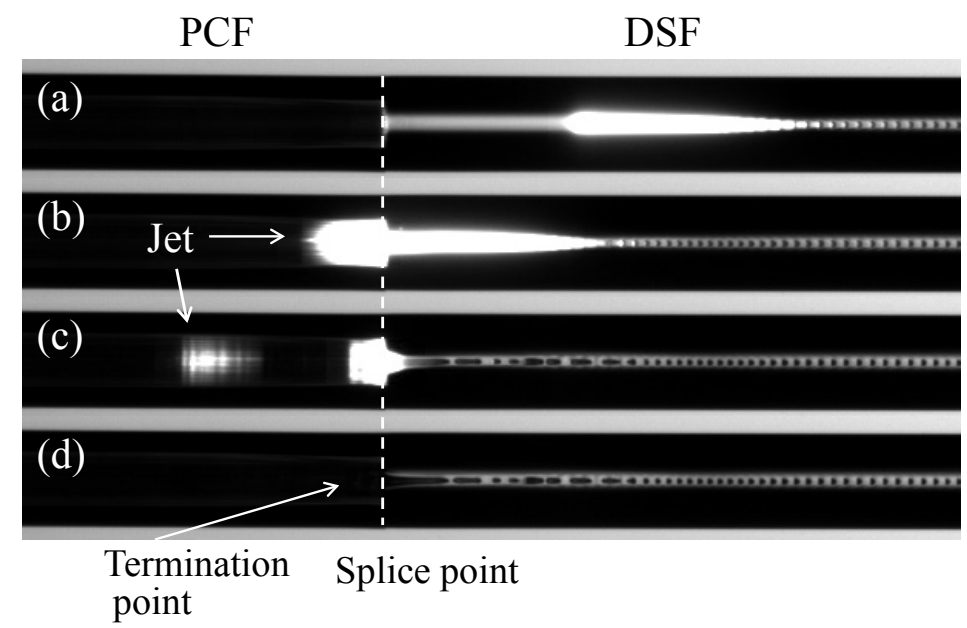

The propagation characteristics of the fiber fuse in HAF depend on the relationship between the diameter of an inscribed circle linking the air holes $c$ and $D_{\text {melted }}[18,20]$. When $c$ was much larger than

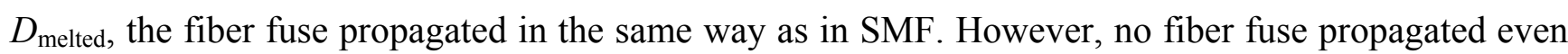
at an input power of $15.6 \mathrm{~W}$, when $c$ was much smaller than $D_{\text {melted }}$ [20]. In the present HAF, we observed no fiber fuse even at a maximum input power of $18.0 \mathrm{~W}(4.4 \mathrm{~W}$ at $1480 \mathrm{~nm}$ and $13.6 \mathrm{~W}$ at $1550 \mathrm{~nm}$ ). Since the $c$ value of the HAF was $17.1 \mu \mathrm{m}, c$ was much smaller than the measured $D_{\text {melted }}$ value of $24.6 \mu \mathrm{m}$ in the $\mathrm{SMF}$.

Figure 4. Dynamics of fiber fuse termination near the splice point between HAF and SMF.

(a)-(d) were obtained at intervals of $0.15 \mathrm{~ms}$. Exposure time was $2.3 \mu \mathrm{s}$.

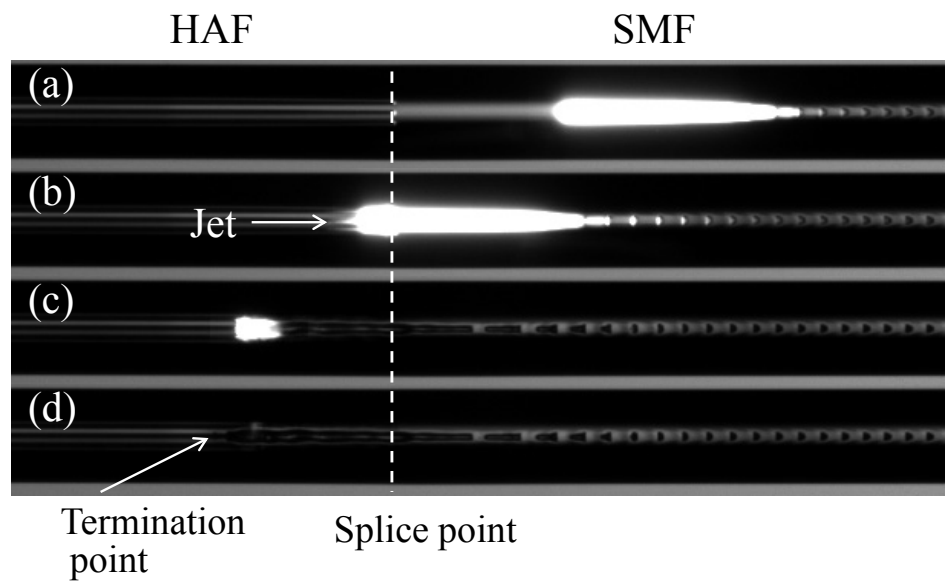

Figure 4 shows the dynamics of fiber fuse termination at a splice point between an SMF and a HAF. Figure $4 \mathrm{a}-\mathrm{d}$ were obtained at intervals of $0.15 \mathrm{~ms}$. The input power was $18.0 \mathrm{~W}$. The exposure time of the high-speed camera was $2.3 \mu \mathrm{s}$. We used an SMF instead of a DSF as fiber 2 to reduce a splice loss between HAF and fiber 2 caused by the mode field mismatch. As shown in Figure 4a, the optical discharge propagated in the SMF leaving periodic voids. When the front of the optical discharge reached the splice point, the front shape of the optical discharge changed and we observed a jet that 
penetrated the air holes of the HAF, as shown in Figure 4b. After $0.15 \mathrm{~ms}$, the optical discharge became small as shown in Figure $4 \mathrm{c}$ and then terminated as shown in Figure 4d.

Since we observed no fiber fuse propagation in this HAF for an input power below $18.0 \mathrm{~W}$, we can consider that the propagation threshold of a fiber fuse in the HAF is above 18.0 W. Therefore, we found that the propagation threshold of a fiber fuse in PCF and HAF can exceed $18 \mathrm{~W}$, which is more than 10 times that in conventional SMF. Since the fuse threshold power in conventional single-mode fibers is proportional to the mode field diameter (MFD) [9], we consider that such a high threshold could not be obtained with a method that uses the MFD expansion of conventional single-mode fibers. High threshold powers in PCF and HAF are considered to be obtained because a fiber fuse in PCF and HAF is terminated by a jet of high temperature fluid penetrating the air holes. A photonic bandgap fiber can also be expected to have a high propagation threshold for the fiber fuse phenomenon. However, we consider that a further loss reduction in the photonic bandgap fiber is desirable if we are to employ it as an optical transmission line [39].

\section{Failures in Bent Fibers}

\subsection{Failures at Bends in SMF and Fiber Fuse Generation}

Failures at bends in an optical fiber can be caused by light leaking from the core when the fiber is accidentally bent tightly with a high power input. The absorption of the leaked light in the fiber coating will heat it and result in failures at bends. Failures in a tightly bent fiber were examined by $180^{\circ}$ bend of conventional SMF with a transparent UV coating in the similar way described in reference 32 . We used a $1480 \mathrm{~nm}$ CW Raman fiber laser as a high power optical source. The power coupled into the sample fiber was $9 \mathrm{~W}$. We observed two failure regimes, "R1 failure" and "R2 failure" [29]. Figure 5 shows an example of an R1 failure. The bend diameter was $11 \mathrm{~mm}$. After 24 min's exposure, the color of the coating started to change as a result of oxidization. As shown in Figure 5a, combustion occurred after 1.5 h's exposure. The coating around the combustion point became black from further oxidization and the coating carbonized. Simultaneously the fiber deformed and the coating was burned, as shown in Figure 5b. It is considered that the fiber temperature exceeds $1100{ }^{\circ} \mathrm{C}$ in an $\mathrm{R} 1$ failure, which causes the fiber to deform under stress [29]. An R1 failure is serious because it causes deformation of the glass leading to complete attenuation of the light and it can also cause the coating to burn, as shown in Figure $5 b$.

Figure 5. Example of R1 failure in SMF at a bend diameter of $11 \mathrm{~mm}$. Input power was 9 $\mathrm{W}$ at $1480 \mathrm{~nm}$. (a) After 1.5 hours' exposure and (b) almost simultaneous with (a).
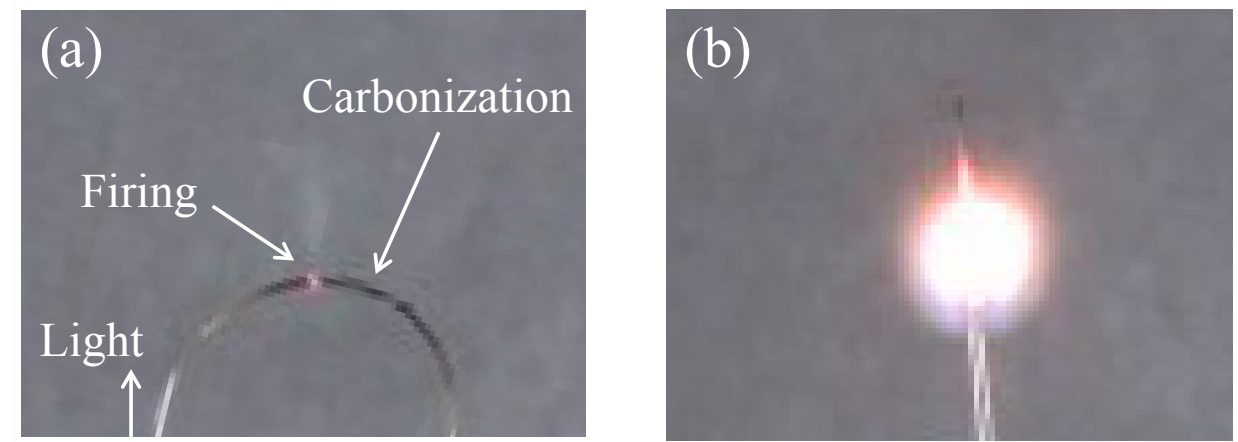
When the bend diameter was $17 \mathrm{~mm}$, we observed an R2 failure after ten hours' exposure, as shown in Figure 6. The coating changed color as a result of oxidization and carbonization. Furthermore, part of the coating vaporized, as shown in Figure 6. Although we observed no deformation of the glass as observed with the R1 failure shown in Figure 5b, handling or local disturbance after an R2 failure will cause the fiber to fracture because the coating is vaporized or burned off [29]. The bend diameter corresponding to the R1/R2 boundary in conventional SMF was 16-17 mm when the input power was $9 \mathrm{~W}$ at a wavelength of $1480 \mathrm{~nm}$.

It is important to note that we observed the generation of a fiber fuse when the bend diameter was $13 \mathrm{~mm}$, which corresponded to an R1 failure. After two hours' exposure, the coating burned and there was simultaneous deformation of the glass. About 1/30 second after the deformation of the glass, the fiber fuse appeared and propagated toward the light source. Therefore, we found that an R1 failure is dangerous because a fiber fuse can be generated under certain conditions in the R1 failure regime and this will seriously damage the optical transmission system. We will examine the details of the conditions under which a fiber fuse is generated in the R1 failure regime in the near future.

Figure 6. R2 failure in $\mathrm{SMF}$ at a bend diameter of $17 \mathrm{~mm}$. Input power was $9 \mathrm{~W}$ at $1480 \mathrm{~nm}$.

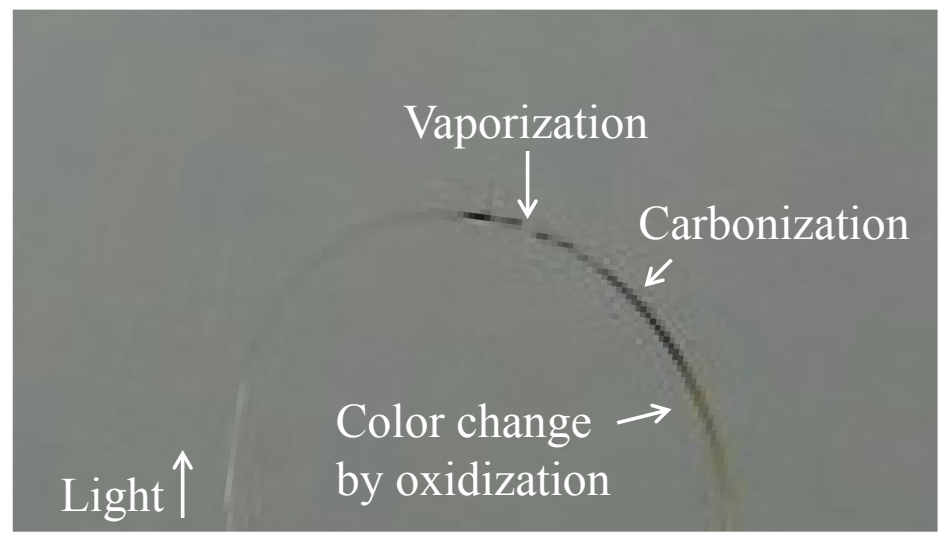

\subsection{Solutions for the Problem of Failures at Bends}

Two kinds of solutions have been reported for the problem of failures at bends in optical fibers. One involves improving the coating to avoid failures. The other is to use optical fibers with low bending losses to reduce the light that leaks from the core as a result of the bending.

First, there are two methods for improving the coating. One is to use a polymer coating with a lower refractive index than the cladding [31]. By using this method, we can restrict the leaked light to the glass portion and avoid heating the coating. The leaked light can also be restricted to the glass portion by employing a second glass cladding with a lower refractive index [32]. The other method involves the use of a special coating with a higher damage threshold. A special coating has been reported that realized (1) a reduction in the coating absorption and stress, and (2) an increase in the homogeneity to increase the threshold of laser induced damage at a wavelength of $2140 \mathrm{~nm}$ for medical applications [34]. This coating had a lower refractive index than that of silica and it had a high resistance to failures at bends at $2140 \mathrm{~nm}$. 
Several kinds of optical fibers with low bending losses have already been developed, namely, PCFs [25,40], HAFs [27], trench-index-profile fibers [41], and nano-engineered fibers [42]. Recent progress on fiber to the home (FTTH) has accelerated the development and standardization of bend-insensitive fibers. ITU-T has produced recommendations on bend-insensitive fibers for use in access networks [43]. Therefore, with a view to avoiding failures at bends, we should use the low bending-loss optical fibers described above with the improved coating that has high resistance to failures at bends.

\section{Conclusion}

We examined optical fibers suitable for avoiding such problems as the fiber fuse phenomenon and failures at bends with a high power input. We found that the threshold power of fiber fuse propagation in PCF and HAF can exceed $18 \mathrm{~W}$, which is more than 10 times that in conventional SMF. This high threshold power in PCF and HAF is considered to be caused by a jet of high temperature fluid penetrating the air holes. We showed examples of two kinds of failures at bends (R1 and R2 failures) in conventional SMF when the input power was $9 \mathrm{~W}$ at a wavelength of $1480 \mathrm{~nm}$. We also observed the generation of a fiber fuse under certain conditions in the R1 failure regime. Solutions for the problems of the failures at bends were described. We showed that one of the solutions is to use optical fibers with a low bending loss such as PCF and HAF. Therefore, we consider PCF and HAF to be attractive optical fibers as a solution for the problems posed by the fiber fuse phenomenon and failures at bends with a high power input.

\section{References}

1. Kashyap, R.; Blow, K.J. Observation of catastrophic self-propelled self-focusing in optical fibers. Electron. Lett. 1988, 24, 47-49.

2. Hand, D.P.; Russell, P.S.J. Solitary thermal shock waves and optical damage in optical fibers: the fiber fuse. Optics Letters 1988, 13, 767-769.

3. Percival, R.M.; Sikora, E.S.R.; Wyatt, R. Catastrophic damage and accelerated aging in bent fibres caused by high optical powers. Electron. Lett. 2000, 36, 414-416.

4. Takenaga, K.; Omori, S.; Goto, R.; Tanigawa, S.; Matsuo, S.; Himeno, K. Evaluation of high-power endurance of bend-insensitive fibers. In Proceedings of the Optical Fiber Communication/National Fiber Optic Engineers Conference, San Diego, CA, USA, 24-28 February 2008.

5. Morioka, T. New generation optical infrastructure technologies: "EXAT Initiative" towards 2020 and beyond. In Proceedings of the OptoElectronics and Communication Conference, Hong Kong, China, 13-17 July 2009.

6. Driscoll, T.J.; Calo, J.M.; Lawandy, N.M. Explaining the optical fuse. Optics Letters 1991, 16, 1046-1048.

7. Davis, D.D.; Mettler, S.C.; DiGiovanni, D.J. Experimental data on the fiber fuse. In Proceedings of SPIE; Bennett, H.E., Guenther, A.H., Kozlowski, M.R., Newnam, B.E., Soileau, M.J., Eds.; SPIE: Washington, WA, USA, 1996; Volume 2714, pp. 202-210. 
8. Atkins, R.M.; Simpkins, P.G. Track of a fiber fuse: A Rayleigh instability in optical waveguides. Optics Letters 2003, 28, 974-976.

9. Nishimura, N.; Seo, K.; Shiino, M.; Yuguchi, R. Study of high-power endurance characteristics in optical fiber link. In Proceedings of the Optical Amplifiers and Their Applications, Otaru, Japan, 6 July 2003.

10. Dianov, E.M.; Bufetov, I.A.; Frolov, A.A.; Chamorovsky, Y.K.; Ivanov, G.A.; Vorobjev, I.L. Fiber fuse effect in microstructured fibers. IEEE Photonic. Technol. Lett. 2004, 16, 180-181.

11. Shuto, Y.; Yanagi, S.; Asakawa, S.; Kobayashi, M.; Nagase, R. Fiber fuse phenomenon in step-index single-mode optical fibers. IEEE J. Quantum Electron. 2004, 40, 1113-1121.

12. Dianov, E.M.; Bufetov, I.A.; Frolov, A.A. Destruction of silica fiber cladding by the fuse effect. Optics Letters 2004, 29, 1852-1854.

13. Yakovlenko, S.I. On reasons for strong absorption of light in an optical fibre at high temperature. Quantum Electron. 2004, 34, 787-789.

14. Dianov, E.M.; Fortov, V.E.; Bufetov, I.A.; Efremov, V.P.; Rakitin, A.E.; Melkumov, M.A.; Kulish, M.I.; Frolov, A.A. Temperature of optical discharge under action of laser radiation in silica-based fibers. In Proceedings of the European Conference and Exhibition on Optical Communication, Glasgow, UK, 25-29 September 2005.

15. Todoroki, S. Origin of periodic void formation during fiber fuse. Opt. Express 2005, 13, 6381-6389.

16. Yakovlenko, S.I. Mechanism for the void formation in the bright spot of a fiber fuse. Laser Phys. 2006, 16, 474-476.

17. Akhmediev, N.; Russell, P.S.J.; Taki, M.; Soto-Crespo, J.M. Heat dissipative solitons in optical fibers. Phys. Lett. A 2008, 372, 1531-1534.

18. Takenaga, K.; Tanigawa, S.; Matsuo, S.; Fujimaki, M.; Tsuchiya, H. Fiber fuse phenomenon in hole-assisted fibers. In Proceedings of the European Conference and Exhibition on Optical Communication, Brussels, Belgium, 21-25 September 2008.

19. Takara, H.; Masuda, H.; Kanbara, H.; Abe, Y.; Miyamoto, Y.; Nagase, R.; Morioka, T.; Matsuoka, S.; Shimizu, M.; Hagimoto, K. Evaluation of fiber fuse characteristics of hole-assisted fiber for high power optical transmission systems. In Proceedings of the European Conference and Exhibition on Optical Communication, Vienna, Austria, 20-24 September 2009.

20. Hanzawa, N.; Kurokawa, K.; Tsujikawa, K.; Matsui, T.; Nakajima, K.; Tomita, S.; Tsubokawa, M. Suppression of fiber fuse propagation in hole assisted fiber and photonic crystal fiber. J. Lightwave Technol. 2010, 28, 2115-2120.

21. Hanzawa, N.; Kurokawa, K.; Tsujikawa, K.; Takenaga, K.; Tanigawa, S.; Matsuo, S.; Tomita, S. Observation of a propagation mode of a fiber fuse with a long-period damage track in hole-assisted fiber. Optics Letters 2010, 35, 2004-2006.

22. Hand, D.P.; Birks, T.A. Single-Mode tapers as "fiber fuse" damage circuit-breakers. Electron. Lett. 1989, 25, 33-34.

23. Yanagi, S.; Asakawa, S.; Kobayashi, M.; Shuto, Y.; Nagase, R. Fiber fuse terminator. In Proceedings of the Pacific Rim Conference on Lasers and Electro-Optics, Taipei, Taiwan, 15-19 December 2003. 
24. Abedin, K.S.; Nakazawa, M.; Miyazaki, T. Backreflected radiation due to a propagating fiber fuse. Opt. Express 2009, 17, 6525-6531.

25. Birks, T.A.; Knight, J.C.; Russell, P.S.J. Endlessly single-mode photonic crystal fiber. Optics Letters 1997, 22, 961-963.

26. Kurokawa, K.; Nakajima, K.; Tsujikawa, K.; Yamamoto, T.; Tajima, K. Ultra-Wideband WDM transmission over PCF. J. Lightwave Technol. 2009, 27, 1653-1662.

27. Nakajima, K.; Hogari, K.; Zhou, J.; Tajima, K.; Sankawa, I. Hole-Assisted fiber design for small bending and splice losses. IEEE Photon. Technol. Lett. 2003, 15, 1737-1739.

28. Logunov, S.L.; Derosa, M.E. Effect of coating heating by high power in optical fibres at small bend diameters. Electron. Lett. 2003, 39, 897-898.

29. Sikora, E.S.R.; McCartney, D.J.; Farrow, K.; Davey, R. Reduction in fibre reliability due to high optical power. Electron. Lett. 2003, 39, 1043-1044.

30. Kurokawa, K.; Fukai, C.; Zhou, J.; Nakajima, K.; Tajima, K.; Hogari, K.; Sankawa, I. High power tolerance of optical fiber cable. In Proceedings of the OptoElectronics and Communication Conference/Conference on Optical Internet, Yokohama, Japan, 12-16 July 2004.

31. Davis, I.M.; Glaesemann, G.S.; Ten, S.; Winningham, M.J. Optical fibers resilient to failure in bending under high power. In Proceedings of the European Conference and Exhibition on Optical Communication, Glasgow, UK, 25-29 September 2005.

32. Chien, C.-K.; Clark, D.A.; Glaesemann, G.S.S. Coating failure of bent fiber under high power laser. In Proceedings of the International Wire \& Cable Symposium and Conference, Providence, RI, USA, 13-16 November 2005.

33. Bigot-Astruc, M.; Sillard, P.; Gauchard, S.; Leroux, P.; Brandon, E. Analysis of coating temperature increase in fibers under high power and tight bending. In Proceedings of the Optical Fiber Communication Conference, Anaheim, CA, USA, 17-22 March 2006.

34. Sun, X.; Lie, J.; Hokansson, A. Study of optical fiber damage under high optical power at 2140 nm. In Proceedings of SPIE; Gannot, I., Ed.; SPIE: Washington, WA, USA, 2007; Volume 6433, pp. 9-15.

35. Sikora, E.S.R.; McCartney, D.J.; Wright, J.V. Impact of coating ageing on susceptibility to high-power damage at fibre bends. Electron. Lett. 2007, 43, 208-210.

36. Matsui, T.; Nakajima, K.; Kurokawa, K.; Tajima, K.; Shiraki, K.; Sankawa, I. Temperature-Increase characteristics in bent hole-assisted fiber under high power. J. Lightwave Technol. 2007, 25, 1231-1237.

37. Logunov, S.L.; Chien, C.-K.; Clark, D.A. High power laser damage of standard and bend resistant fibres. Electron. Lett. 2009, 45, 1019-1020.

38. Kurokawa, K.; Hanzawa, N.; Tsujikawa, K.; Tmita, S. Hole-Size dependence of fiber fuse propagation in hole-assisted fiber (HAF). In Proceedings of the Microoptics Conference, Sendai, Japan, 30 October-2 November 2011.

39. Roberts, P.J.; Couny, F.; Sabert, H.; Mangan, B.; Williams, D.P.; Farr, L.; Mason, M.W.; Tomlinson, A.; Birks, T.A.; Knight, J.C.; Russell, P.S.J. Ultimate low loss of hollow-core photonic crystal fibres. Opt. Express 2005, 13, 236-244.

40. Sorensen, T.; Broeng, J.; Bjarklev, A.; Knudsen, E.; Barkou Libori, S.E. Macro-Bending loss properties of photonic crystal fibre. Electron. Lett. 2001, 37, 287-289. 
41. Himeno, K.; Matsuo, S.; Guan, N.; Wada, A. Low-Bending-Loss single-mode fibers for fiber-to-the-home. J. Lightwave Technol. 2005, 23, 3494-3499.

42. Li, M.-J.; Tandon, P.; Bookbinder, D.C.; Bickham, S.R.; McDermott, M.A.; Desorcie, R.B.; Nolan, D.A.; Johnson, J.J.; Lewis, K.A.; Englebert, J.J. Ultra-Low bending loss single-mode fiber for FTTH. J. Lightwave Technol. 2009, 27, 376-382.

43. ITU-T Recommendation G.657, 2nd ed.; ITU-T: Geneva, Switzerland, 2009.

(C) 2012 by the authors; licensee MDPI, Basel, Switzerland. This article is an open access article distributed under the terms and conditions of the Creative Commons Attribution license (http://creativecommons.org/licenses/by/3.0/). 